\title{
Monitoring Hydrogen Evolution Reaction Intermediates of Transition Metal Dichalcogenides via Operando Raman Spectroscopy
}

\author{
Shaohui Guo, Yaohua Li, Songwei Tang, Yuanyuan Zhang, Xuanhua Li, ${ }^{\star}$ Ana Jorge
} Sobrido, Maria-Magdalena Titirici, and Bingqing Wei*

A deeper understanding of the water-splitting hydrogen evolution reaction (HER) mechanism during photocatalytic processes is crucial for the rational design of efficient photocatalysts. In particular, the HER mechanism promoted by multielement hybrid structures remains extremely challenging and elusive. Herein, an in situ photoelectrochemical/Raman measurement system is employed to monitor the HER mechanism of hybrid nanostructures under realistic working conditions via operando Raman spectra and linear-sweep voltammetry curves. As a proof of concept, tunable composition transition metal dichalcogenides MoS2xSe2(1-x) nanosheets are used as a model photocatalyst to unveil the corresponding photocatalytic mechanism. The spectroscopic studies reveal that hydrogen atoms can be adsorbed to active sulfur and selenium atoms via intermediate species formed during the photocatalytic process. More importantly, the studies demonstrate that an exponential relationship exists between the number of reactive electrons and the Raman intensity of intermediate species, which can serve as a guideline to directly evaluate the HER performance in photocatalysts by comparing the Raman intensities of the intermediate species. As a simple, intuitive, and general analytical method, the designed operando Raman measurement approach provides a new tool for elucidating catalytic reaction mechanisms in a realistic and complex environment; and strategically improving $\mathrm{H}_{2}$ production performance of multielement photocatalysts.

\section{Introduction}

The hydrogen evolution reaction (HER) via photocatalytic water splitting is a promising alternative to fossil fuel production amidst the increasing energy demands.[1-5] As such, developing efficient photocatalytic water splitting methods for high performance HER processes has been the subject of increased research efforts. [6-9] Hydrogen evolution occurs via a two-step process involving adsorption of hydrogen atoms and desorption of a hydrogen molecule.[10] During this transformation, intermediate species are formed and react on the surface of the photocatalysts.[11] Thus, a thorough understanding of this surface process is needed to further the development of these reactions.

Various approaches have been employed to illustrate the intricate photocatalytic water-splitting $\mathrm{H}_{2}$ production reactions. For instance, density functional theory (DFT) calculations have been utilized in combination with a crystalline structure, optical, and electronic structural features of the photocatalysts to elucidate the reaction mechanisms.[12-16] Moreover, scanning transmission electron microscopy,[17] infrared spectroscopy,[18,19] and nuclear magnetic resonance spectroscopy[20,21] have been used to probe photocatalytic transformations. Although these techniques can simulate or detect interactions between adsorbed water molecules and photocatalysts, most of them obtain their data in the departure reaction condition (not reaction condition). Moreover, many of these techniques operate under ideal conditions (e.g., under ultrahigh vacuum). Recently, operando X-ray absorption spectroscopy has been used to identify the active sites of photocatalysts and the reaction processes under real conditions.[22] However, this technique is relatively complex and, therefore, not possible for all research needs. Comparatively, the operando Raman technique is a useful method to analyze the structural transformation of photocatalysts under real HER reaction condition.[23,24] Nevertheless, a few simple photocatalysts have been studied via the Raman method, such as Pt and CoSez; and the research is only involved in the relative primitive photocatalytic reaction process.[23,24] The advance in the design of new heterostructures- and multielement-based catalysts requires a deep study about the complex reaction processes they are involved in order to enhance photocatalytic performance.[25-28]

Herein, we employ a linear-sweep voltammetry (LSV)-operando Raman spectroscopy measurement system to 1) reveal the photocatalytic HER intermediates of multielement photocatalysts under real reaction conditions. 2) Furthermore, we establish an exponential function relationship between the quantity of reactive electrons (i.e., photocatalytic performance) and the Raman intensity of intermediate species, which can serve as a guideline to evaluate the HER performance directly and strategically improve $\mathrm{H}_{2}$ production performance of multielement photocatalysts. Considering the excellent photocatalytic performance of layered transition metal dichalcogenides, 
we select the MoS2xSe2(1-x) nanosheets ( $x$ gradually decreases from 1 to 0 ) as the photocatalyst. As shown in Figure 1, a three-electrode photoelectrochemical system has been used to carry out and monitor the catalytic $\mathrm{H}_{2}$ evolution reaction under light illumination. The LSV plots obtained from the photoelectrochemical system have been used to record the catalytic reaction. Simultaneously, Raman measurements on the working electrode containing the photocatalyst in the fluorine-doped tin oxide (FTO) glass were carried out to characterize the surface reaction transformations of the samples. By combining operando Raman spectra and LSV plots, the photocatalytic HER mechanism can be revealed in the $\mathrm{H}_{2}$ production process under real conditions.

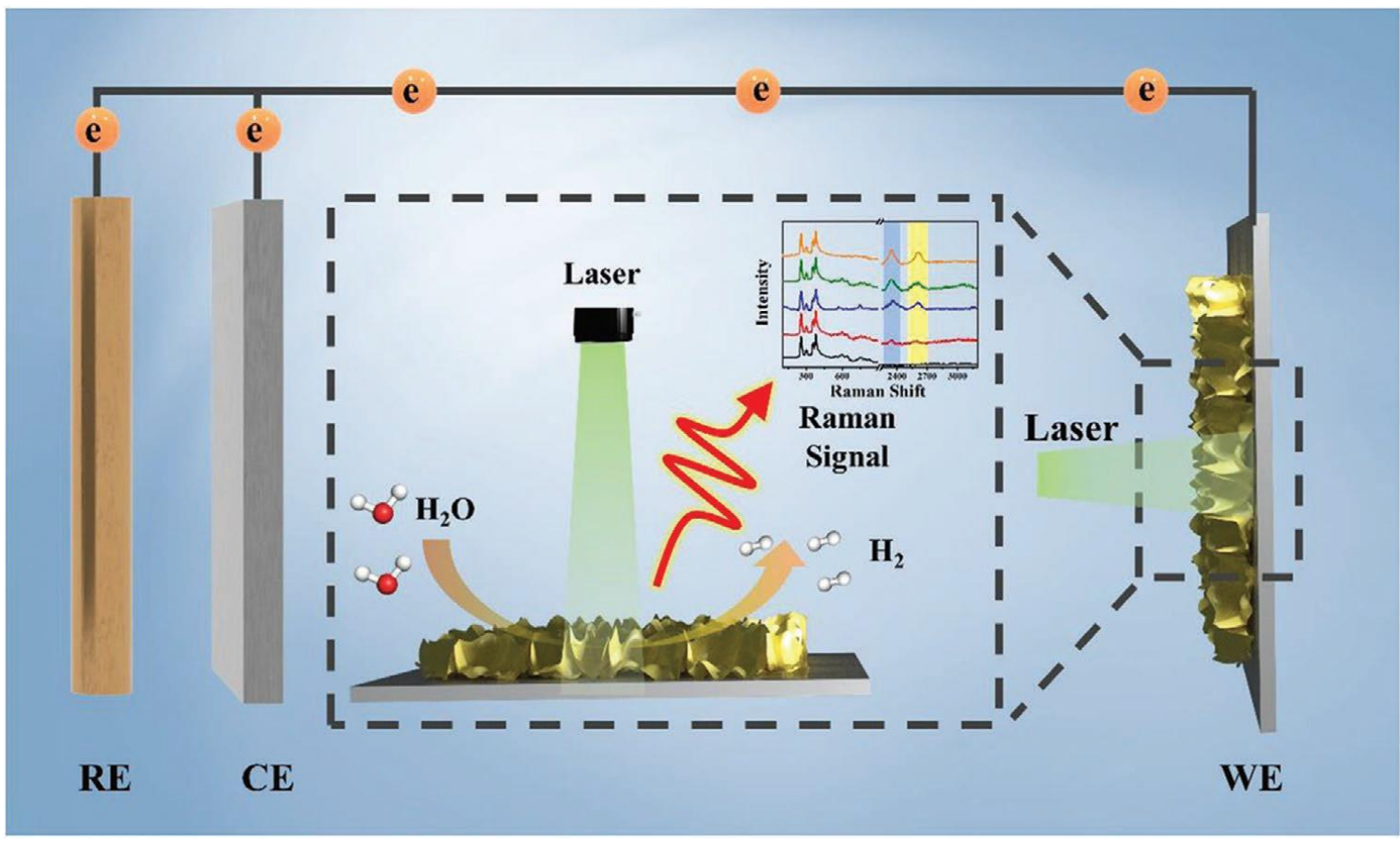

Figure 1. The schematic of the photoelectrochemical system with LSV-Raman analysis. The measured material is MoS2xSe2(1-x). The reference electrode, counter electrode, and working electrode are termed as RE, CE, and WE, respectively.

\section{Results and Discussion}

In the current work, the vertical $\operatorname{MoS}_{2 x} \mathrm{Se}_{2}(1-x)$ nanosheets are prepared to expose more edge active atoms for exploring the HER mechanism. Figure 2 displays the morphology of the MoS2xSe2(1-x) nanosheets from $x=1$ to $x$ $=0$ (i.e., MoS2, MoS1.4Se0.6, MoS0.9Se1.1, MoS0.4Se1.6, and MoSe2, in which the atomic ratio is derived from energydispersive X-ray spectroscopy [EDS], which is also agreeable with the X-ray photoelectron spectroscopy [XPS] spectra; Figure S1, Supporting Information). All of the photocatalyst materials exhibit similar nanoflake morphologies with the visible lines representing their standing edges. The EDS mapping of the ternary

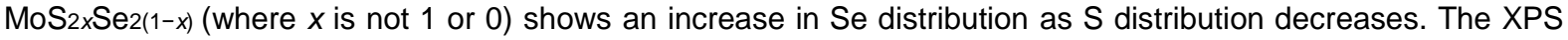
spectra, related X-ray diffraction patterns, ultraviolet-visible absorption spectra, and Raman spectra have been

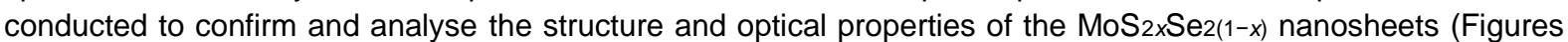
S1-S4, Supporting Information). Interestingly, the S-Se bonding is detected in the MoS0.4Se1.6 sample (Figure S1d, Supporting Information), which indicates that the presence of a relatively high number of Se atoms in the structure leads to interaction with the $S$ atoms. 


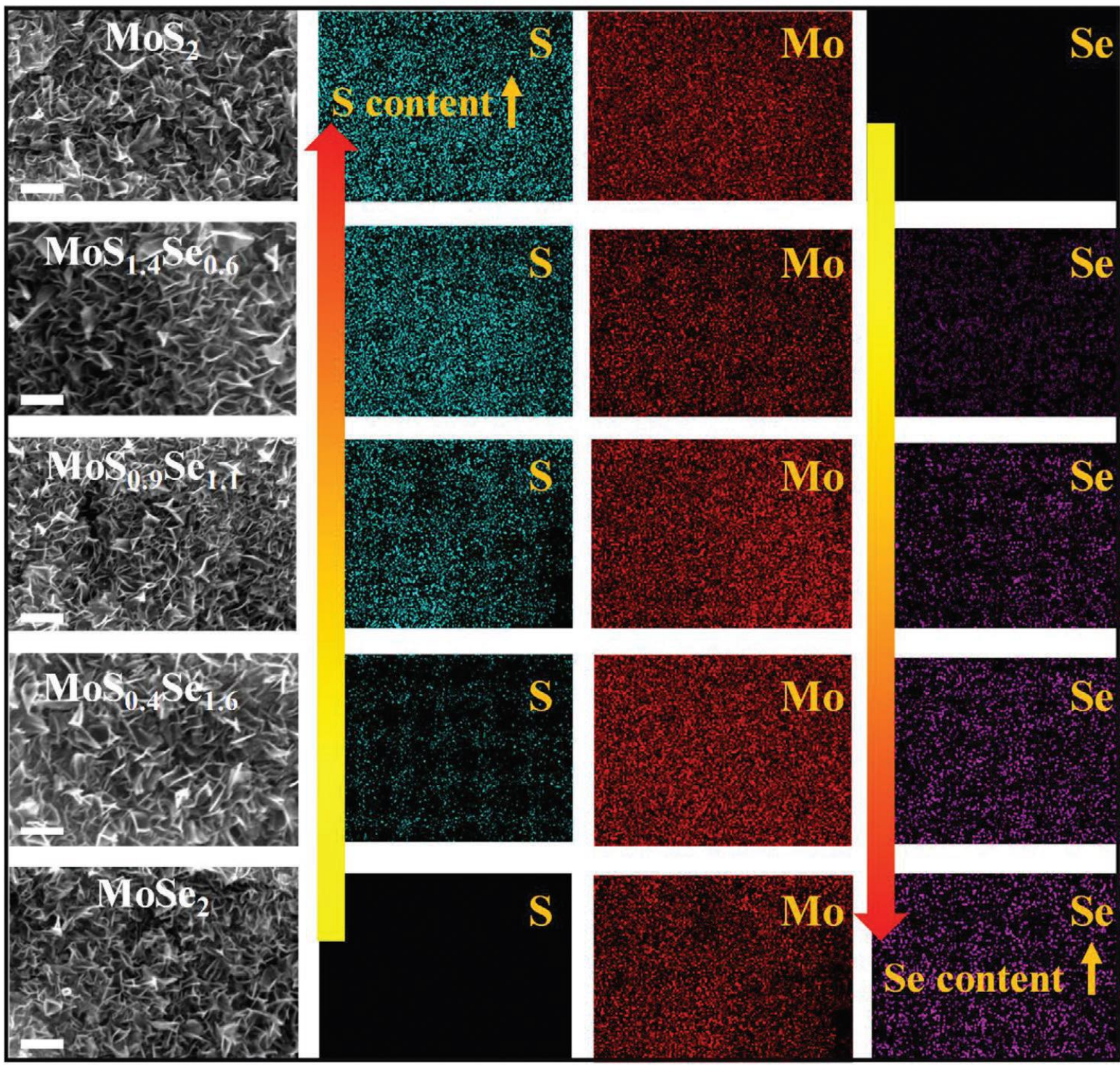

Figure 2. The SEM images of $\operatorname{MoS}_{2 x} \operatorname{Se}_{2(1-x)}(x$ decreases from 1 to 0$)$ and the corresponding EDS mapping. The scale bar is 500 $\mathrm{nm}$.

Figure 3a displays the LSV curve of the binary MoS2 sample under light illumination. To avoid the influence of the bubble formation from the gas evolution reaction, we performed the LSV-operando Raman spectroscopy measurements in the voltage window from 0.05 to $-0.15 \mathrm{~V}$. No current exists at $0.05 \mathrm{~V}$, as there is no photoinduced electron transport occurring at this voltage. Below $0.05 \mathrm{~V}$, the appearance of photocurrent can be easily observed. As the voltage increases in value, the current density also increases, exhibiting a current density of approximately $0.33 \mathrm{~mA} \mathrm{~cm}-2$ at $-0.15 \mathrm{~V}$ for the MoS2 sample. The Raman spectra from five voltage points in the LSV plots show two obvious peaks at 380 and $408 \mathrm{~cm}-1$, which are assigned to E2g(Mo-S) and A1g(Mo-S), respectively (Figure 3b).[29] Interestingly, as the voltage varies from 0.05 to $-0.15 \mathrm{~V}$, a new peak at $2584 \mathrm{~cm}-1$ appears, showing higher intensity for the voltage at $-0.15 \mathrm{~V}$. The observed frequency, $2584 \mathrm{~cm}-1$, matches well with the $\mathrm{S}-\mathrm{H}$ stretching vibration,[30] which strongly suggests that intermediate MoS2-H species are formed during the HER process. It also indicates that the $\mathrm{H}$ atom is bonded to $\mathrm{S}$ atoms during the photocatalytic reaction. The intermediate MoS2-H species are also confirmed by DFT theory simulation about the hydrogen adsorption of MoS2 (Figure S5, Supporting Information), which is agreeable with the experimental result.

The current density of the binary MoSez also increases along with variable voltages from 0.05 to $-0.15 \mathrm{~V}$ (Figure 3c). At $-0.15 \mathrm{~V}$, the current density reaches approximately $0.49 \mathrm{~mA} \mathrm{~cm}-2$, a value greater than that of MoS2. The related operando Raman spectra of MoSe2 are displayed in Figure 3d. Two peaks at 257 (A1g(Mo-Se)) and $287 \mathrm{~cm}-1$ (E2g(Mo-Se)) are observed.[31] In addition, a new peak at $2365 \mathrm{~cm}-1$ with increased peak intensity appears as the voltage varied from 0.05 to $-0.15 \mathrm{~V}$. Similar to the $\mathrm{S}-\mathrm{H}$ vibration band in the case of MoS2, this Raman peak is consistent with $\mathrm{Se}-\mathrm{H}$ stretching vibrations, $[32,33]$ indicating that the $\mathrm{H}$ atoms are adsorbed to the active Se atoms to 
form intermediate MoSe2-H species here. The deduction is also verified by DFT simulation about the hydrogen adsorption of MoSe2 (Figure S5, Supporting Information). Through this simple and intuitive operando Raman technique, the catalytic reaction intermediate species can be identified.

(a)

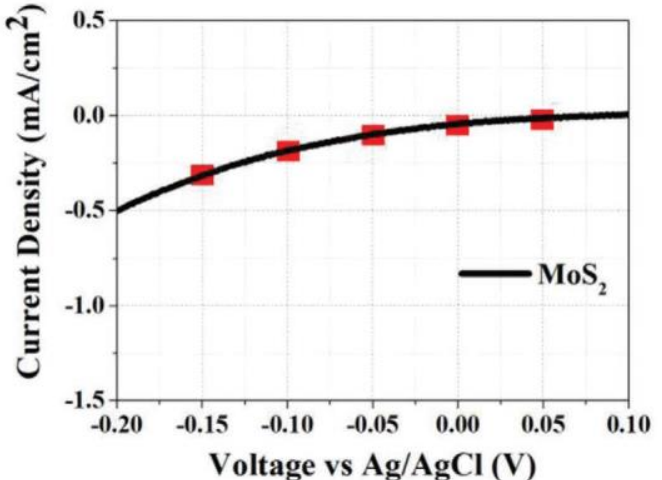

(c)

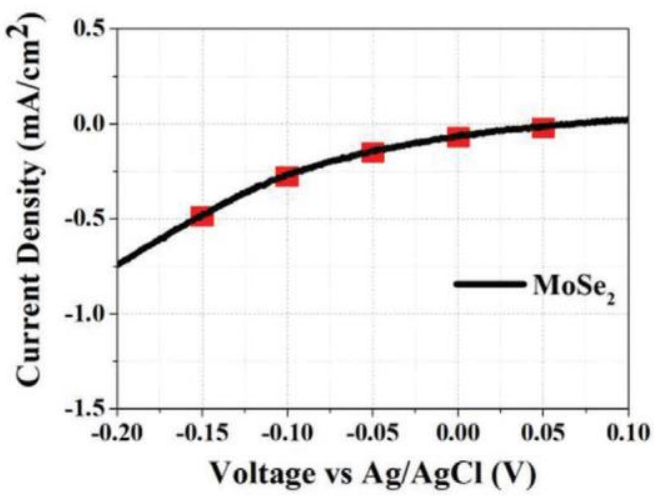

(e)

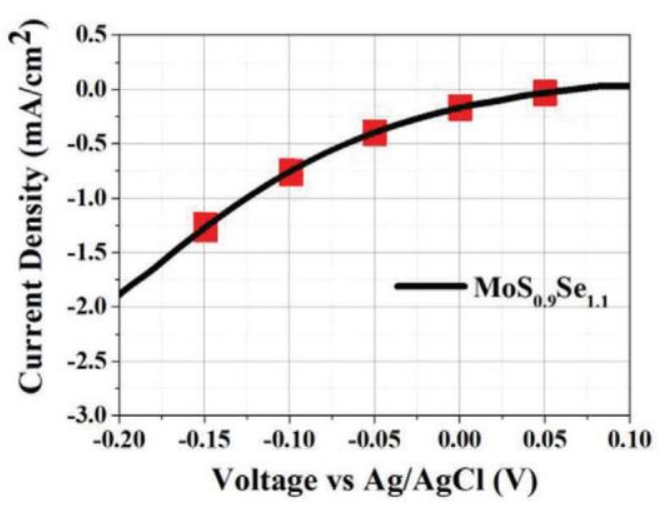

(b)

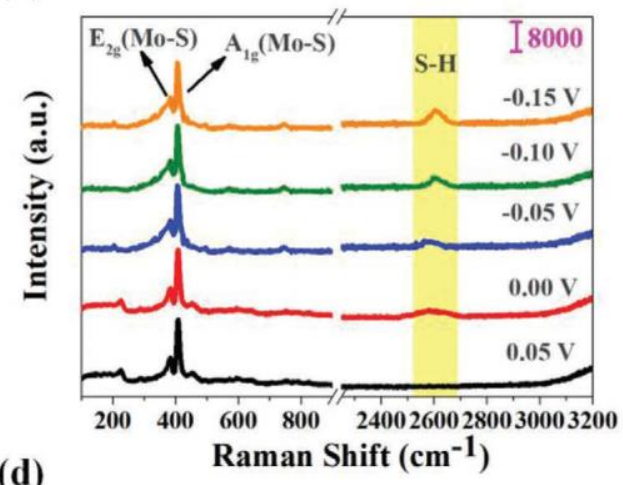

(d)

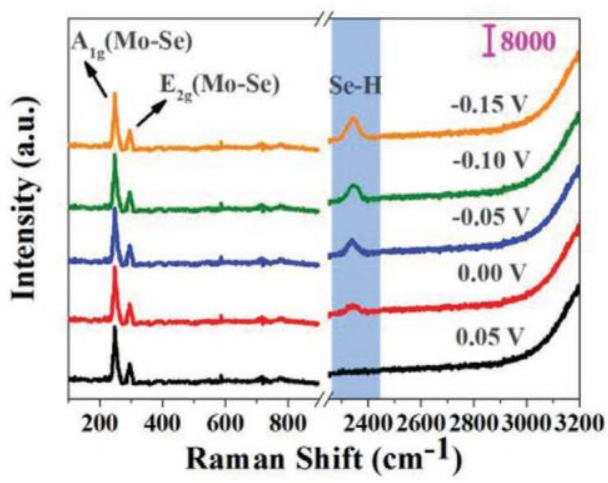

(f)

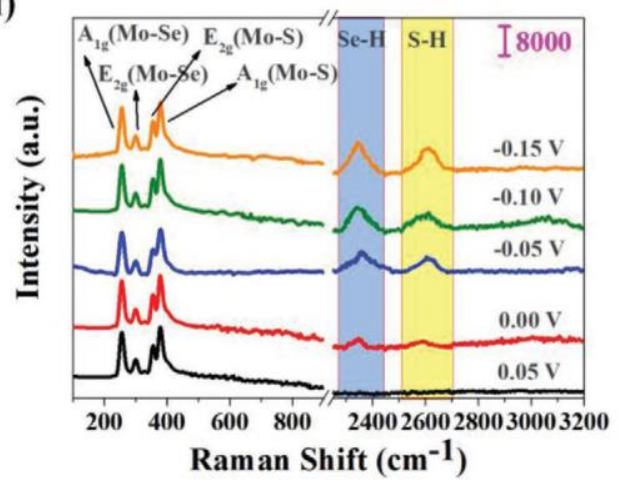

(g)

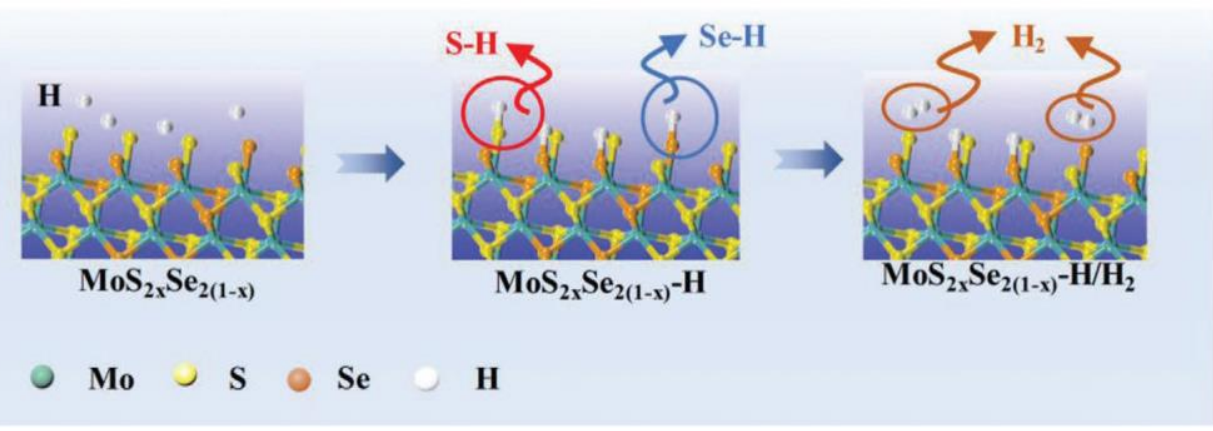

Figure 3. a) LSV curve of the sample $\mathrm{MoS}_{2}$, b) operando Raman spectra of the sample MoS2 in the voltage of $0.05,0.00,-0.05$, -0.10 , and $-0.15 \mathrm{~V}, \mathrm{c}) \mathrm{LSV}$ curve of the sample MoSe2, d) operando Raman spectra of the sample MoSez in the voltage of 0.05 , $0.00,-0.05,-0.10$, and $-0.15 \mathrm{~V}$, e) LSV curve of the sample MoSo.9Se $1.1, \mathrm{f}$ ) operando Raman spectra of the sample MoSo.9Se1.1

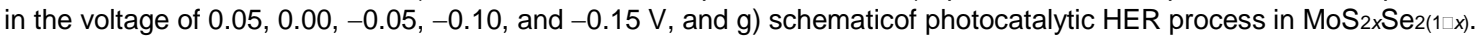


The next step is to assess whether this operando measurement method would be suitable for complex, multielement photocatalysts. For these studies, the ternary MoS $2 x \operatorname{Se} 2(1-x)$ materials (where $x$ is not 1 or 0 ) are used as the photocatalytic materials of choice. The LSV curves and the corresponding operando Raman spectra of the ternary MoS $0.9 S_{1} 1.1$ are displayed in Figure 3e,f. The LSV curves demonstrate that there is no current at $0.05 \mathrm{~V}$ (versus $\mathrm{Ag} / \mathrm{AgCl}$ ), whereas the current density of MoS0.9Se1.1 reached approximately $0.21 \mathrm{~mA} \mathrm{~cm}-2$ at $0.00 \mathrm{~V}$. Varying the voltage further to $-0.15 \mathrm{~V}$, a current density of $1.27 \mathrm{~mA} \mathrm{~cm}-2$ is obtained. These results show that the ternary MoS0.9Se1.1 sample exhibits higher current density than the binary MoS2 or MoSe2 samples, indicating that the addition of an extra element (i.e., either Se or S) in the nanostructures can efficiently promote a photo-induced electron response (the reason will be discussed later). The operando Raman measurements from the MoS0.9Se1.1 sample depict four apparent peaks at 360, 384, 259, and $298 \mathrm{~cm}-1$, owing to the Raman vibration modes of S $\square$ Mo and Se $\square$ Mo (Figure 3f).[34] In addition to these four characteristic Raman peaks, two Raman peaks $\square \mathrm{H}$ Raman

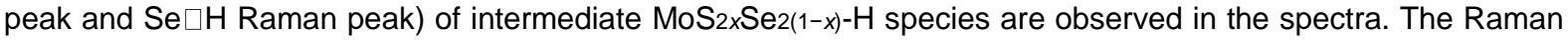
peak intensities of the intermediate species increased together with varying voltage from 0.05 to $-0.15 \mathrm{~V}$. The LSVoperando Raman spectra of the ternary MoS $1.4 \mathrm{Se}_{0.6}$ and MoS $0.4 \mathrm{Se}_{1.6}$ are also carried out and are shown in Figures S6-S8, Supporting Information. Similarly, the S $\square \mathrm{H}$ and Se $\square \mathrm{H}$ Raman stretching vibration peaks are also observed in the intermediate MoS1.4Se0.6- $\mathrm{H}$ and MoS $0.4 \mathrm{Se}_{1.6}-\mathrm{H}$ species. As such, this operando Raman measurement is applicable for studying the HER intermediate species of the complicated, multielement photocatalytic systems.

Based on these experimental data, the HER mechanism of transition metal dichalcogenides MoS $2 x \operatorname{Se} 2(1-x)$ can be discussed (Figure $3 \mathrm{~g}$ ). As for the binary photocatalysts, during the photocatalytic HER process, $\mathrm{H}$ atoms are initially adsorbed to the active $\mathrm{S}$ atoms (or Se atoms) to form intermediate MoS2-H (or MoSe2- $\mathrm{H}$ ) species based on $\mathrm{S}-\mathrm{H}$ bonding (or Se-H bonding) first in MoS2 (or MoSe2); subsequently, the redox reaction occurs to produce hydrogen. This experimental evidence illustrates that the active sites are S atoms or Se atoms in the MoS2 (or MoSe2) rather than Mo atoms. Regarding the complex, multielement photocatalysts, $\mathrm{H}$ atoms from water are initially adsorbed to the active $\mathrm{S}$ atoms and Se atoms to form intermediate MoS2xSe2(1-x)-H species at the MoS2xSe2(1-x) Surface; subsequently, hydrogen desorption occurs to realize $\mathrm{H}_{2}$ production. In other words, the catalytic HER process can be understood through the reaction formulas below:

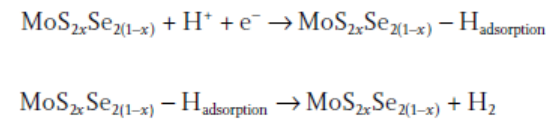

where $\mathrm{H}_{+}$is the hydrogen ions, the MoS2xSe2(1-x)-Hadsorption is the intermediate species and the e-is the electron, which is equivalent to the current density in the LSV curve. Our finding is consistent with the theoretical prediction in previous reports, $[15,16,35]$ which further corroborates that probing the catalytic hydrogen evolution reaction intermediates via operando Raman spectra is valid and sensible.

To further understand the relationship between the intermediate species and the $\mathrm{H}_{2}$ production performance in the reaction, we plot the Raman intensity of the $\mathrm{S}-\mathrm{H}$ and $\mathrm{Se}-\mathrm{H}$ bands in the intermediate MoS2xSe2(1-x)-H species from the $\mathrm{MoS}_{2 x} \mathrm{Se}_{2(1-x)}$ operando Raman spectra against the applied voltage (Figure 4a,b). (Note: the S-H and Se-H intensities have been converted to intensities based on atomic moles and electrochemically active surface areas [Figure S9, Supporting Information] for better comparison, see the Experimental Section). It demonstrates that the trend of the S-H intensity and Se-H intensity among the MoS2xSe2(1-x) operando Raman spectra is consistent with the photocatalytic performance (Figure S6, Supporting Information). Furthermore, we plot the relationship between intermediate species Raman intensity and the photocatalytic performance using applied voltage as a linking bridge, as shown in Figure 4c,d. After the curves have been projected to the current density-intensity plane, we observe that the Raman intensity of intermediate species increases together with the increase of current density. Strictly, the curves in the current density-intensity plane can be fitted. Take MoS0.9Se1.1 as an example. As shown in Figure $4 \mathrm{e}$, the fitting formulas are below:

$$
\begin{aligned}
& y=0.01747 e^{0.00113 x} \\
& Y=0.02395 e^{0.00107 x}
\end{aligned}
$$

where $y$ is the current density, $x$ is the intermediate species Raman intensity. The Equation (3) describes the relationship between the S-H Raman intensity and the current density. Similarly, the Equation (4) describes the relationship between the Se-H Raman intensity and the current density. We observe that the trend between the current density and the Raman intensity of intermediate species in MoS 0.9 Se 1.1 is close to the exponential function 
type. The trend is also applicable to the other $\operatorname{MoS}_{2 x} \operatorname{Se} 2(1-x)$ catalysts, as shown in Figures S10-S13, Supporting Information.

(a)

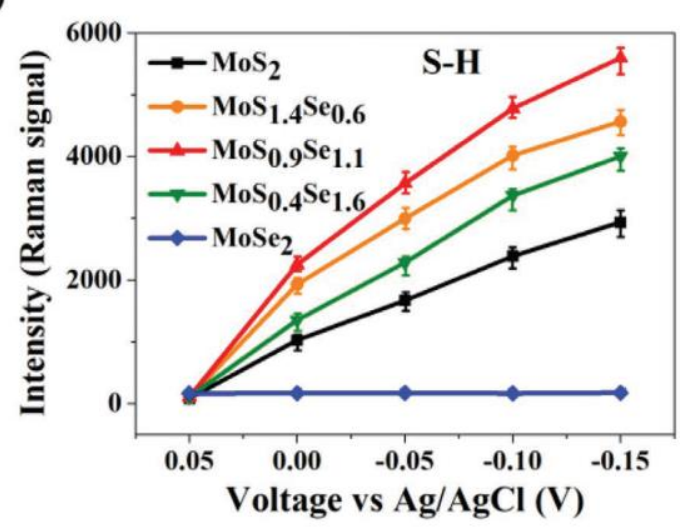

(c)

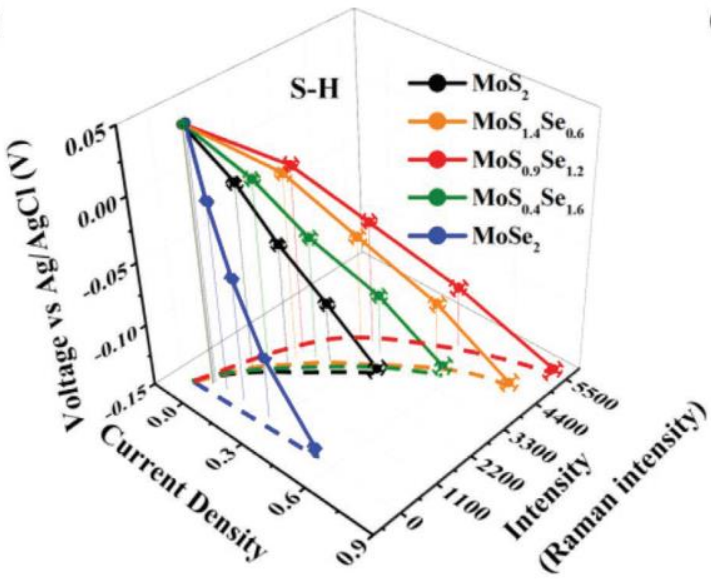

(e)

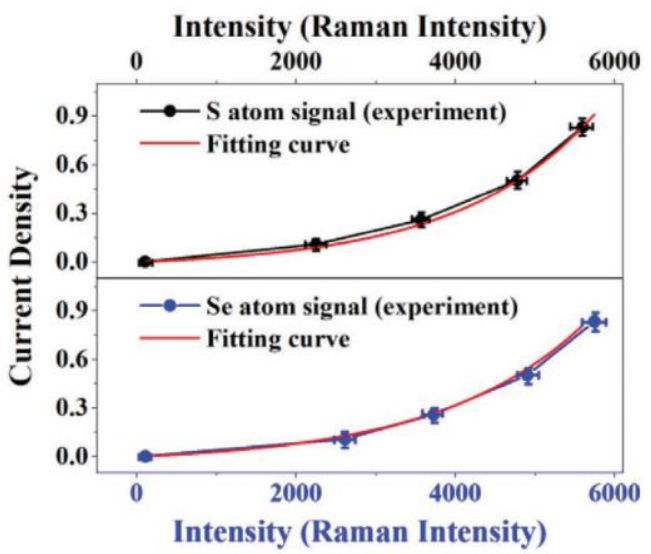

(b)

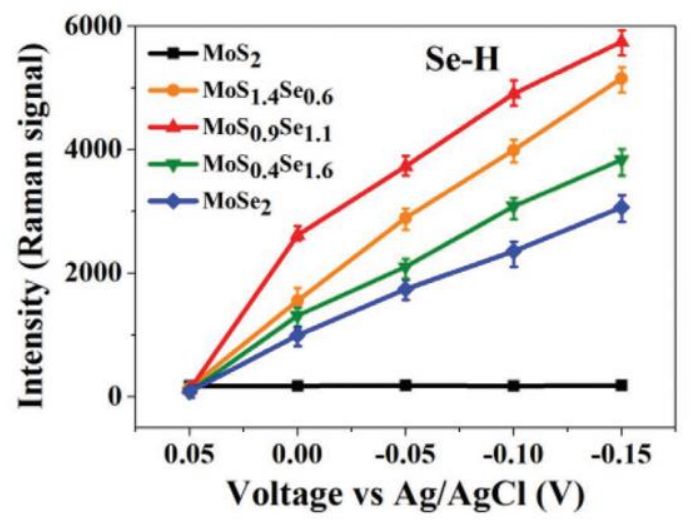

(d)

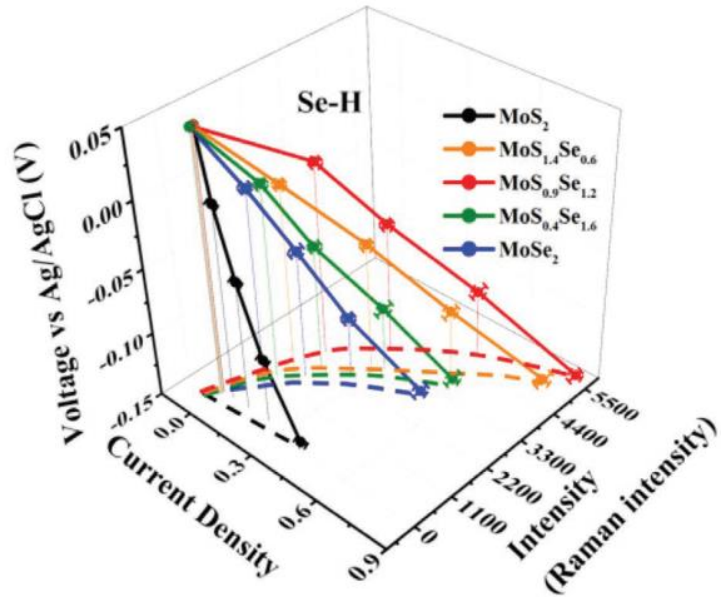

(f)

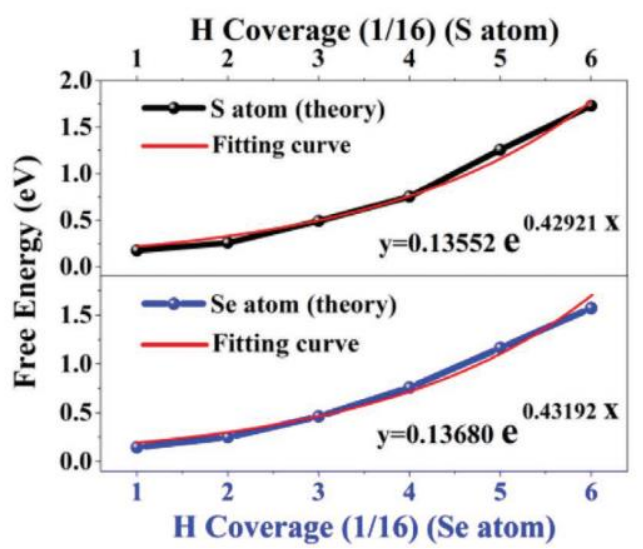

Figure 4. a) S-H stretching vibration intensity from $\operatorname{MoS}_{2 x} \mathrm{Se}_{2(1 \square x)}$ operando Raman spectra, b) Se-H stretching vibration intensity from $\mathrm{MoS}_{2 x} \mathrm{Se}_{2(1-x)}$ operando Raman spectra, c) relationship between current density and S-H stretching vibration intensity using applied voltage as a linking bridge, d) relationship between current density and $\mathrm{Se}-\mathrm{H}$ stretching vibration intensity using applied voltage as a linking bridge, e) the relationship between current density and Raman intensity of intermediate species based on experimental results in MoS0.9Se1.1, and f) the relationship between free energy and $\mathrm{H}$ coverage on single edge atom through DFT calculation in MoS0.9Se1.1.

To further figure out the exponential function relationship, we carry out the DFT calculation to reveal internal causes. The Raman intensity of intermediate species from abscissa in Figure $4 \mathrm{e}$ can be understood as intermediate species quantity; and the intermediate species generally can be known as a group of hydrogen adsorption on the surface of photocatalysts. [36] Based on the description of intermediate species, the $\mathrm{H}$ coverage is considered here, and the $\mathrm{H}$ coverage is defined as the hydrogen adsorption number on the edge active atoms of MoS $2 x \mathrm{Se}_{2}(1-x)$. There are 16 atoms on a single edge, including $8 \mathrm{~S}$ atoms and $8 \mathrm{Se}$ atoms in a supercell from the current theoretical model, 
as shown in Figure S14, Supporting Information. The adsorption of one hydrogen atoms on a supercell is defined to coverage of $1 / 16$, and full coverage of $16 / 16$ means that all atoms on the single edge are loaded by hydrogen atoms. Considering that the free energy change of hydrogen adsorption can be used as an evaluation index to catalytic performance, $[37,38]$ thus the free energy depending on the $\mathrm{H}$ coverage has been calculated (Figure $4 \mathrm{f}$ ). It is easily noticed that free energy increases exponentially versus $\mathrm{H}$ coverage, which is consistent with the above fitting formulas.

Additionally, composition change does a great effect on intermediate species quantity, which is evidenced through the Raman intensity in Figure 4a,b. The hydrogen adsorption process of the MoS2xSe2(1-x) photocatalysts with different composition ratios have been further discussed with DFT calculation. As shown in Figure 5, the hydrogen adsorption configurations in binary and ternary $\operatorname{MoS}_{2 x} \mathrm{Se}_{2(1-x)}$ photocatalysts are intuitively displayed. Comparing to the binary photocatalysts $\left(\mathrm{MoS}_{2}\right.$ and $\mathrm{MoSe}_{2}$ in Figure $5 \mathrm{a}, \mathrm{e}$ ), the hydrogen adsorption ability of the ternary photocatalysts is boosted (Figure $5 b-d$ ). The sample MoSo.9Se1.1 possesses the optimal hydrogen adsorption numbers and forms the most intermediate species among the ternary photocatalysts. In the sample MoSo.4Se1.6, the formation of $\mathrm{S} \square \mathrm{Se}$ bonding weakens the hydrogen adsorption ability, leading to less intermediate species production, which reduces Raman intensity of the intermediate species (Figure 4a,b).

(a)

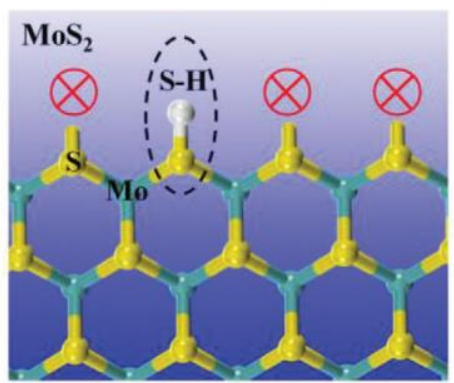

(d)

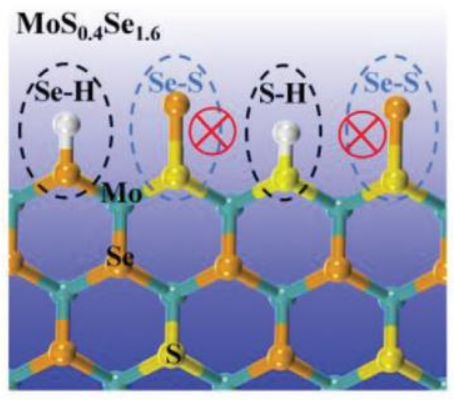

(b)

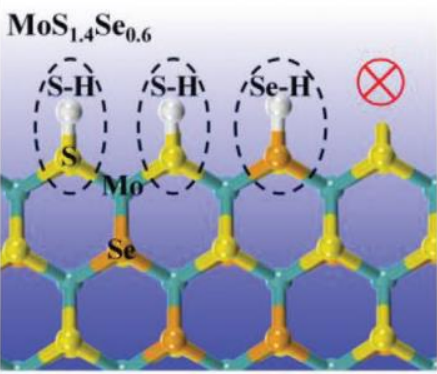

(e)

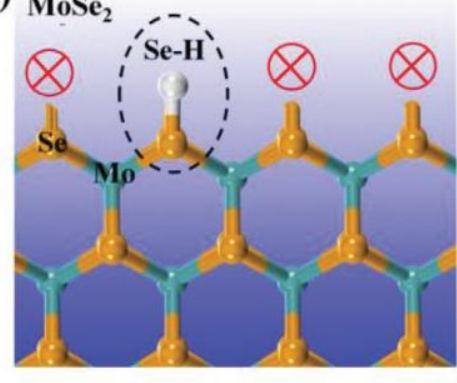

(c)

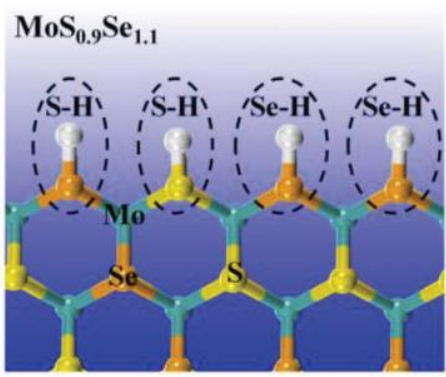

(f)

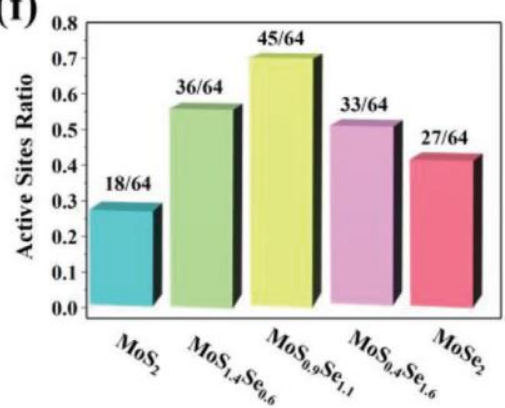

(g)

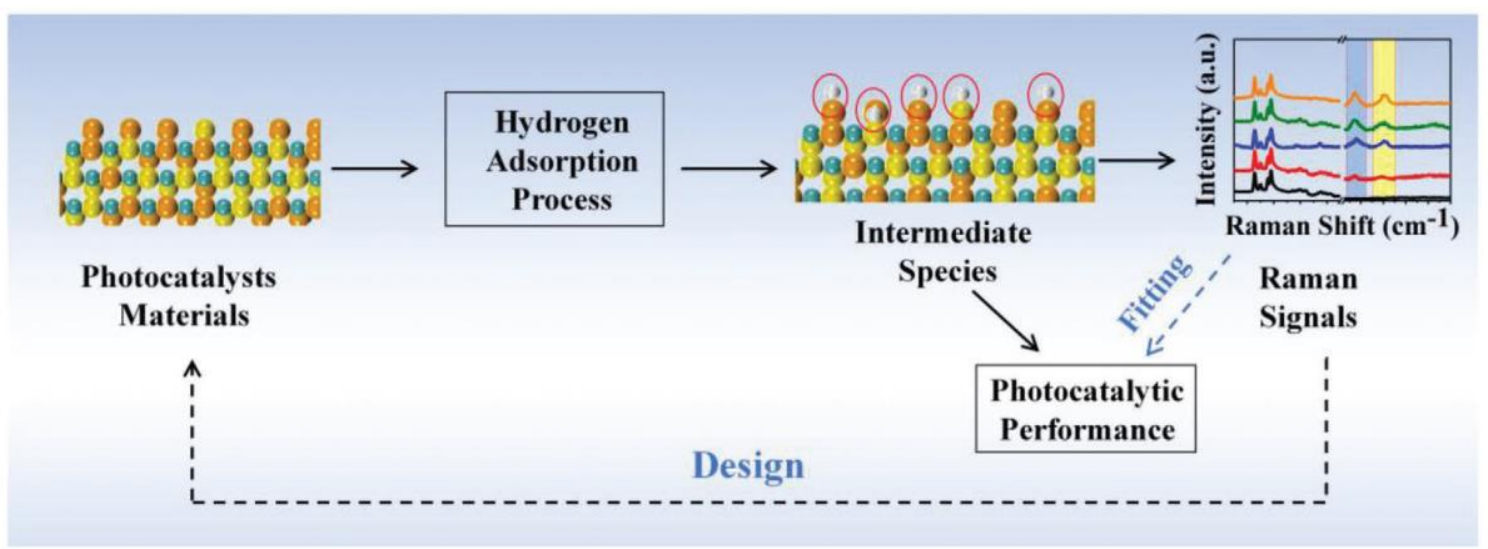

Figure 5. Hydrogen adsorption configuration of HER process in a) MoS2, b) MoS $1.4 \mathrm{Se}_{0.6}$, c) MoSo.9Se1.1, d) MoS $0.4 \mathrm{Se}_{1.6}$, and e) MoSe2, f) active sites ratios of all five samples, and g) the summary of the relationship among photocatalysts materials, hydrogen adsorption process, intermediate species, photocatalytic performance, and Raman signals.

Based on the hydrogen adsorption process, the active sites/total sites ratio has been calculated in Figure $5 f$. The MoS2xSe2(1-x) nanosheets are established with 16 atoms on each edge, and active catalytic sites are chosen 
according to the free energy changes for hydrogen adsorption (see Figures S14 and S15, Supporting Information). As for the binary photocatalysts, the catalytic active sites ratios of the samples MoS2 and MoSe2 are 18/64 and $27 / 64$, respectively. The catalytic active sites ratio of the ternary photocatalysts is increased to $36 / 64$ for the sample MoS 1.4Se0.6. The sample MoS0.9Se1.1 possesses the highest catalytic active sites ratio (45/64), while the catalytic active sites ratio of the sample MoS0.4Se1.6 decreases to 33/64. Thus, the high catalytic active sites ratio of the sample MoS0.9Se1.1 is ideal for forming abundant intermediate species, thus enhancing the photocatalytic activity, which is also consistent with the DFT calculation results about free energy for hydrogen absorption in different samples (Figure S5, Supporting Information).

In Figure 5g, we summarize the relationship among photocatalysts materials, hydrogen adsorption process, intermediate species, photocatalytic performance, and Raman signals. First, the formation of intermediate species is determined by the hydrogen adsorption ability in photocatalyst materials. The high active site ratio is optimal to form more intermediate species. Furthermore, the Raman measurements can detect the Raman signals of intermediate species to supplement the catalytic mechanism. Here, the detection of intermediate species MoS2xSe2(1-x)-H through Raman spectra have shone some light on the HER mechanism for the MoS2xSe2(1-x) photocatalysts. Moreover, an exponential function relationship between the photocatalytic performance (i.e., the quantity of reactive electrons) and the intermediate species evidenced by the operando Raman measurement was revealed. This finding can serve as a guideline to evaluate photocatalytic performance and mechanism by comparing the Raman intensity of intermediate species. For instance, in Figures 3 and 4, the sample MoSo.9Se1.1 shows the strongest Raman intensity of intermediate species, indicating the best photocatalytic performance among $\mathrm{MoS}_{2 x} \mathrm{Se}_{2}(1-x)$ photocatalysts. More importantly, the relationship between the photocatalytic performance and the Raman intensity of intermediate species can function as a guide for the rational design of multielement photocatalysts. By comparing the Raman intensity of intermediate species, we can tune the elemental proportion in the photocatalyst materials to realize better photocatalytic performance.

\section{Conclusion}

In summary, we have developed a remarkably simple, intuitive, and versatile method to probe the photocatalytic HER intermediates of complicated and multielement photocatalysts under realistic reaction conditions using the operando Raman technique. Based on LSV curves and Raman spectra analysis during the HER process, we have demonstrated that hydrogen atoms are initially adsorbed to the active $S$ atoms or Se atoms by chemical bonding $\mathrm{S} \square \mathrm{H}$ and $\mathrm{Se} \square \mathrm{H}$ to form intermediate species in $\operatorname{MoS} 2 x S_{2}(1-x)$, followed by the subsequent redox reaction. In addition, we have established that an exponential function relationship exists between the quantity of reactive electrons and the Raman intensity of intermediate species, which can be used as a guideline to evaluate the $\mathrm{H}_{2}$ production performance of the photocatalysts. The designed operando Raman spectroscopy can also be extended to other photocatalytic reactions where Raman-active intermediate species are formed. Moreover, it provides a new approach to understand the surface science and reaction mechanisms of HER photocatalysts in a real and complex environment and hence, to strategically improve their $\mathrm{H}_{2}$ production performance.

\section{Experimental Section}

Preparation of the Photocatalysts-Ternary MoS2xSe2(1-x) Nanosheets: For the synthesis of the vertical MoS $2 x \operatorname{Se}_{2(1-x)}$ (where $x$ is not equal to 0 or 1) nanosheets, a porcelain boat loaded with $0.5 \mathrm{~g} \mathrm{MoO}_{3}$ powder was placed into the high heating temperature zone of a slender quartz tube. Two additional boats loaded with sulfur and selenium powders were placed at the upstream lowtemperature zone. FTO substrates were placed downstream next to the porcelain boat with the $\mathrm{MoO}_{3}$ powder. Before heating, an $\mathrm{Ar}$ gas flow was introduced into the system at a rate of 100 s.c.c.m. for $20 \mathrm{~min}$. The high heating temperature zone in the furnace was then heated to $800 \square \mathrm{C}$ for $40 \mathrm{~min}$, and the low-temperature zone was simultaneously heated to between 250 and $350 \square \mathrm{C}$. After heating both zones for $40 \mathrm{~min}$, these temperatures were maintained for $10 \mathrm{~min}$, during which time Ar gas was allowed to flow at a rate of 100 s.c.c.m. to transport sulfur and selenium to the substrate. After the growth of the materials, the

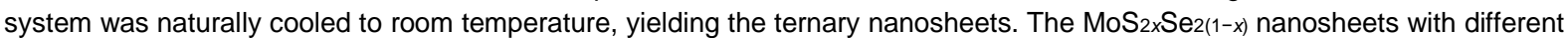
compositions were deposited onto the surface of substrates through tuning different $\mathrm{S} \square \mathrm{Se}$ content ratios in precursors (MoS1.4Se0.6: precursor S $\square$ Se content ratio 5:2, MoS0.9Se1.1: precursor S $\square$ Se content ratio 1:1, and MoSo.4Se1.6: precursor S $\square$ Se content ratio 1:4) and heating temperatures in the low-temperature zone (MoS 1.4 Se0.6 for $250 \square \mathrm{C}$, MoSo.9Se1.1 for $300 \square \mathrm{C}$, and MoS 0.4 Se 1.6 for $350 \square$ C).

Preparation of the Photocatalysts-MoS2 Nanosheets and MoSe2 Nanosheets: The preparation procedures of the MoS 2 and MoSez nanosheets were similar to those of the ternary $\operatorname{MoS}_{2 x} \mathrm{Se}_{2(1-x)}$ nanosheets. For the MoS2 nanosheets, only sulfur powder was placed at the lowtemperature zone, and the heating temperature in the low-temperature region was kept at approximately $220 \square \mathrm{C}$. For the MoSez nanosheets, only selenium powder was placed at the low-temperature zone, and the heating temperature in the low-temperature region was around $380 \square \mathrm{C}$.

Characterization of the Photocatalysts: The morphology of the samples was characterized using SEM (FEI NOVASEM). The absorption properties were measured using an UV-vis spectrophotometer (PerkinElmer Lambda 35 UV-vis). The Raman spectra were measured using a Raman spectrometer (Renishaw inVia) with a $532 \mathrm{~nm}$ laser. The XRD patterns were collected via an Empyrean PA Analytical XRD system using Cu Ka radiation as the reference X-ray illumination at $0.154 \mathrm{~nm}$. XPS spectra were 
collected on a Kratos Axis supra XPS spectrometer. The electrochemically active surface area to each sample was obtained from electrochemical capacitance measurement. The potential was swept from 0.12 to $0.22 \mathrm{~V}$ and back to $0.12 \mathrm{~V}$ with four different scan rates $(10,20,30$, and $40 \mathrm{mV} \mathrm{s}-1)$.

Operando Raman Spectra and Photoelectrochemical Measurements: The photoelectrochemical measurements were carried out by a three-electrode reactor, using the nanostructured materials as the working electrode, a Pt plate as the counter electrode, and $\mathrm{Ag} / \mathrm{AgCl}$ (internal electrolyte: saturated $\mathrm{KCl}$ solution) as the reference electrode. A $0.5 \mathrm{~m} \mathrm{Na} \mathrm{SO}_{4}$ solution was used as the electrolyte for the photoelectrochemical studies. The LSV curves were collected through an electrochemical workstation, Chenhua 760 , with a bias voltage set from 0.10 to $-0.20 \mathrm{~V}$. The scan rate was $0.002 \mathrm{~V} \mathrm{~s}-1$. Then, the obtained currents were normalized based on the electrochemically active surface area to each sample. The photocatalysts were illuminated by the light

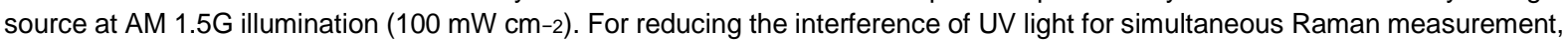
a UV-cut filter was applied. During the photoelectrochemical measurements, the Raman spectra from all of the photocatalysts were collected at the same interval voltage to eliminate the extra influence induced through bias voltage (the measurement voltage was $0.05,0.00,-0.05,-0.10$, and $-0.15 \mathrm{~V})$ under the laser irradiation with same light intensity $(5 \mathrm{~mW})$. The laser wavelength was $532 \mathrm{~nm}$, and the exposure time was $5 \mathrm{~s}$. For each sample, five Raman spectra at random positions on the substrate were collected and then averaged them. The Raman intensities of the intermediate species have been converted to the normalized intensities based on atomic moles and electrochemically active surface areas, which could impact the Raman signals. Specifically, the molar quantity of photocatalysts was calculated through the photocatalysts mass; and the molar quantity of $S$ atom and Se atom were calculated based on the atomic ratio and photocatalyst molar quantity. Then, the modified intensity of intermediate species could be obtained via combining measured Raman intensity and atomic molar quantity. After that, the obtained Raman intensity was further normalized based on the electrochemically active surface area to each sample. The transferred intensity could be used to simply indicate the amount of the intermediate species.

Theoretical Calculation: DFT with the plane-wave-pseudopotential method[39,40] was performed. The Vienna Ab-initio Simulation Package code[41] on the basis of the generalized gradient approximation of the Perdew-Burke-Ernzerhof $[42]$ formula was adopted for all calculations. An energy cut-off of $450 \mathrm{eV}$ was used. The convergence threshold for the residual force was set to $0.01 \mathrm{eV}$ $\AA-1$, while the convergence tolerance of energy during structure relaxation was 10-5 eV. Besides, the Brillouin zone was sampled by the Monkhorst-Pack method[43] with a separation of about $0.03 \AA$. The vacuum space of $20 \AA$ was used to separate neighboring slabs in the $z$-direction. A $15 \AA$ of vacuum was considered in the $y$-direction so that each layer was isolated. The differential hydrogen adsorption energy $\Delta E H$ could be described by the following equation:[44]

$$
\Delta E_{\mathrm{H}}=E_{n \mathrm{H}^{*}}-E_{(n-1) \mathrm{H}^{*}}-\frac{1}{2} E_{\mathrm{H}_{2}}
$$

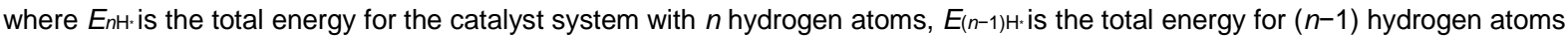
adsorbed on the catalyst. $\mathrm{H}_{2}$ is the energy of the hydrogen molecule in the gas phase. From the differential hydrogen adsorption energy, the free energy $(\Delta G)$ Ho $\cdot$ for hydrogen adsorption could be calculated by Equation (6):

$$
\Delta C_{H^{*}}^{0}=\Delta E_{H}+\Delta E_{\text {ZPE }}-T \Delta S_{H}
$$

(6)

where $\Delta E$ ZPE is the difference in zero-point energy of hydrogen between the adsorbed state and the gas phase, and $\Delta S_{H}$ is the entropy difference between the adsorbed hydrogen and the hydrogen gas. The nanosheets were established like nano-rectangle with 16 atoms on each edge, as shown in Figures S14 and S15, Supporting Information. Regarding the active sites/total sites ratio calculation, the active sites/ total sites ratio was obtained on the basis of free energy change for the adsorption of hydrogen on the edges of transition metal Dichalcogenides $\mathrm{MoS}_{2 x} \mathrm{Se}_{2(1-x)}$ nanosheets.

\section{Supporting Information}

Supporting Information is available from the Wiley Online Library or from the author.

\section{Acknowledgements}

This research was supported by the Basic Research Fund for Free Exploration in Shenzhen (Grant No. JCYJ20180306171402878), the Shaanxi International Cooperation Project (2020KWZ-018), the Project of Shaanxi Young Stars in Science and Technology (2017KJXX-18), and the Fundamental Research Funds for the Central Universities (3102019ghxm003, 3102019JC005, 3102019ghjd001). The authors thank the members from the Analytical and Testing Center of Northwestern Polytechnical University for the help of SEM and XPS characterization.

\section{Conflict of Interest}

The authors declare no conflict of interest.

\section{Keywords}

multielement photocatalysts, operando Raman spectroscopy, photocatalytic reaction intermediates, transition metal Dichalcogenides

[1] Z. Wang, C. Li, K. Domen, Chem. Soc. Rev. 2019, 48, 2109.

[2] D. Voiry, H. S. Shin, K. P. Loh, M. Chhowalla, Nat. Rev. Chem. 2018, 2, 0105.

[3] Z. H. Hu, Z. T. Wu, C. Han, J. He, Z. H. Ni, W. Chen, Chem. Soc. Rev. 2018, 47, 3100.

[4] X. H. Li, S. H. Guo, W. Li, X. G. Ren, J. Su, Q. Song, A. J. Sobrido, B. Q. Wei, Nano Energy 2019, 57, 388. 
[5] Y. Goto, T. Hisatomi, Q. Wang, T. Higashi, K. Ishikiriyama, T. Maeda, Y. Sakata, S. Okunaka, H. Tokudome, M. Katayama, S. Akiyama, H. Nishiyama, Y. Inoue, T. Takewaki, T. Setoyama, T. Minegishi, T. Takata, T. Yamada, K. Domen, Joule 2018, 2 , 509.

[6] D. B. Li, X. Y. Li, S. M. Chen, H. Yang, C. D. Wang, C. Q. Wu, Y. A. Haleem, S. Duan, J. L. Lu, B. H. Ge, P. M. Ajayan, Y. Luo, J. Jiang, L. Song, Nat. Energy 2019, 4, 512.

[7] Z. Lian, M. Sakamoto, Y. Kobayashi, N. Tamai, J. Ma, T. Sakurai, S. Seki, T. Nakagawa, M. W. Lai, M. Haruta, H. Kurata, T. Teranishi, ACS Nano 2019, 13, 8356.

[8] G. Liao, Y. Gong, L. Zhang, H. Gao, G. J. Yang, B. Fang, Energy Environ. Sci. 2019, 12, 2080.

[9] J. N. Tiwari, A. M. Harzandi, M. Ha, S. Sultan, C. W. Myung, H. J. Park, D. Y. Kim, P. Thangavel, A. N. Singh, P. Sharma, S. S. Chandrasekaran, F. Salehnia, J. W. Jang, H. S. Shin, Z. Lee, K. S. Kim, Adv. Energy Mater. 2019, 9, 1900931.

[10] X. Y. Chia, A. Y. S. Eng, A. Ambrosi, S. M. Tan, M. Pumera, Chem. Rev. 2015, 115, 11941.

[11] C. L. Tan, Z. C. Lai, H. Zhang, Adv. Mater. 2017, 29, 1701392.

[12] C. F. Fu, X. J. Wu, J. L. Yang, Adv. Mater. 2018, 30, 1802106.

[13] D. Wang, Z. P. Liu, W. M. Yang, ACS Catal. 2018, 8, 7270.

[14] S. W. Cao, H. Li, T. Tong, H. C. Chen, A. C. Yu, J. G. Yu, H. M. Chen, Adv. Funct. Mater. 2018, 28, 1802169.

[15] A. Travert, H. Nakamura, R. A. van Santen, S. Cristol, J. F. Paul, E. Payen, J. Am. Chem. Soc. 2002, 124, 7084.

[16] Q. Ding, B. Song, P. Xu, S. Jin, Chem 2016, 1, 699.

[17] T. Daio, I. Narita, S. Nandy, T. Hisatomi, K. Domen, K. Suganuma, Chem. Phys. Lett. 2018, 706, 564

[18] M. Zhang, M. de Respinis, H. Frei, Nat. Chem. 2014, 6, 362.

[19] J. G. Highfield, M. H. Chen, P. T. Nguyen, Z. Chen, Energy Environ. Sci. 2009, 2, 991.

[20] A. S. Cattaneo, D. C. Villa, S. Angioni, C. Ferrara, R. Melzi, E. Quartarone, P. Mustarelli, Energy Environ. Sci. 2015, 8, 2383.

[21] X. L. Wang, W. Q. Liu, Y. Y. Yu, Y. H. Song, W. Q. Fang, D. X. Wei, X. Q. Gong, Y. F. Yao, H. G. Yang, Nat. Commun. 2016, 7,1 .

[22] L. L. Cao, Q. Q. Luo, W. Liu, Y. K. Lin, X. K. Liu, Y. J. Cao, W. Zhang, Y. Wu, J. L. Yang, T. Yao, S. Q. Wei, Nat. Catal. 2019, 2,134 .

[23] I. Ledezma-Yanez, O. Daz-Morales, M. C. Figueiredo, M. T. M. Koper, ChemElectroChem 2015, 2, 1612.

[24] Y. Zhu, H. Chen, C. Hsu, T. Lin, C. Chang, S. Chang, L. Tsai, H. M. Chen, ACS Energy Lett. 2019, 4, 987.

[25] S. Xiao, W. Dai, X. Liu, D. Pan, H. Zou, G. Li, G. Zhang, C. Su, D. Zhang, W. Chen, H. Li, Adv. Energy Mater. 2019, 9 , 1900775

[26] X. Xin, Y. R. Song, S. H. Guo, Y. Z. Zhang, B. L. Wang, J. K. Yu, X. H. Li, Appl. Catal., B 2020, 269, 118773.

[27] S. Wang, Z. Li, Y. Zhang, X. Liu, J. Han, X. Li, Z. Liu, S. Liu, W. C. H. Choy, Adv. Funct. Mater. 2019, $29,1900417$.

[28] D. Li, E. J. Podlaha, Nano Lett. 2019, 19, 3569.

[29] S. H. Guo, X. H. Li, X. G. Ren, L. Yang, J. M. Zhu, B. Q. Wei, Adv. Funct. Mater. 2018, 28, 1802567.

[30] Y. L. Deng, L. R. L. Ting, P. H. L. Neo, Y. J. Zhang, A. A. Peterson, B. S. Yeo, ACS Catal. 2016, 6, 7790.

[31] D. Q. Zeng, P. Y. Wu, W. J. Ong, B. S. Tang, M. D. Wu, H. F. Zheng, Y. Z. Chen, D. L. Peng, Appl. Catal., B 2018, $233,26$. [32] J. Kretzschmar, N. Jordan, E. Brendler, S. Tsushima, C. Franzen, H. Foerstendorf, M. Stockmann, K. Heim, V. Brendler, Dalton Trans. 2015, 44, 10508.

[33] X. Zhang, W. Xu, Y. Wang, S. Jiang, F. A. Gorelli, E. Greenberg, V. B. Prakapenka, A. F. Goncharov, Phys. Rev. B 2018, $97,064107$.

[34] H. Li, X. Duan, X. Wu, X. Zhuang, H. Zhou, Q. Zhang, X. Zhu, W. Hu, P. Ren, P. Guo, L. Ma, X. Fan, X. Wang, J. Xu, A. Pan, X. Duan, J. Am. Chem. Soc. 2014, 136, 3756.

[35] P. D. Tran, T. V. Tran, M. Orio, S. Torelli, Q. D. Truong, K. Nayuki, Y. Sasaki, S. Y. Chiam, R. Yi, I. Honma, J. Barber, V. Artero, Nat. Mater. 2016, 15, 640.

[36] W. Lorenz, J. Electroanal. Chem. Interfacial Electrochem. 1989, 265, 159.

[37] X. Fan, S. Wang, Y. An, W. Lau, J. Phys. Chem. C 2016, 120, 1623.

[38] J. Greeley, T. F. Jaramillo, J. Bonde, I. B. Chorkendorff, J. K. Norskov, Nat. Mater. 2006, 5, 909.

[39] G. Kresse, D. Joubert, Phys. Rev. B 1999, 59, 1758.

[40] P. E. Blochl, Phys. Rev. B 1994, 50, 17953.

[41] G. Kresse, J. Furthmuller, Comput. Mater. Sci. 1996, 6, 15.

[42] J. P. Perdew, K. Burke, M. Ernzerhof, Phys. Rev. Lett. 1996, 77, 3865.

[43] H. J. Monkhorst, J. D. Pack, Phys. Rev. B 1976, 13, 5188.

[44] B. Hinnemann, P. G. Moses, J. Bonde, K. P. Jorgensen, J. H. Nielsen, S. Horch, I. Chorkendorff, J. K. Norskov, J. Am. Chem. Soc. 2005, 127, 5308 . 